\title{
Use of AO PSF models for the Study of Resolved Stellar Populations
}

\author{
A. Deep ${ }^{1, a}$, G. Fiorentino ${ }^{2}$, L. Jolissaint ${ }^{1}$, and E. Tolstoy ${ }^{2}$ \\ 1 Leiden Observatory, University of Leiden \\ 2 Kapteyn Institute, University of Groningen
}

\begin{abstract}
The full scientific exploitation of AO images to study resolved stellar populations is still in a nascent stage. This requires pushing to the faint limits and carrying out deep and accurate crowded field photometry and astrometry. The main complexity of AO images is that the correction is never perfect and the PSF always has a complex residual halo around the central core which contains a large fraction of the light. The variations in seeing over short periods of time and anisoplanatism effects create strongly time dependent effects in the PSF. The aim of this study is primarily to test the ability of current photometric packages to be able to cope with a AO PSFs in a number of straightforward but scientifically important examples.
\end{abstract}

\section{Introduction}

It is hoped that in the future, using an Extremely Large Telescope, the detailed resolved stellar populations of Virgo Elliptical galaxies can be studied in enough detail to determine accurate star formation histories. Here we present an initial exploration of the technical requirements to achieve this goal. We create simulations assuming most of the parameters such as background, pixel scale, read-noise, throughput etc what might be expected from the E-ELT instrument MICADO (Multi-AO Imaging Camera for Deep Observations) which is a Phase A study at ESO. MICADO is primarily intended to work with MAORY (a Multi-Conjugate AO system also currently a Phase A study at ESO) but it will also contain a SCAO module for initial operations.

\section{Simulation and photometry}

All the simulations for this document have been made using the IRAF package artdata (artificial data). Table 1 and Table 2 give the MICADO parameters used for simulations. The exposure time has been taken to be 1 hour for each simulation. For the simulations we have used either a $3 \times 3 \operatorname{arcsec~FoV,}$ which corresponds to $1000 \times 1000$ pixels on the detector, or a $0.75 \times 0.75 \operatorname{arcsec}$ FoV, which is $250 \times$ 250 pixels. We stick to a one hour exposure as a representative image depth to determine the generic effects of crowding, and photometric errors.

Two different adaptive optics models have been considered in this study, MCAO and SCAO. The MCAO PSFs are provided by MAORY consortium ([5]) and the SCAO PSFs have been simulated using analytic code PAOLA ([3]). In Figure 1 we show the simulated image of a small region in an old galaxy at $18 \mathrm{Mpc}$ using these PSFs. The I-band image shown there, and the corresponding Ks band image, were analysed using the photometry package Starfinder (developed by E. Diolaitti [2]). The PSF for doing photometry was in each case extracted directly from the simulated field and not assumed $a$ priori. We also carried out a similar comparison with the more commonly used DAOPHOT/Allstar ([6]). Since it returned very similar results to Starfinder, here we only discuss the Starfinder results. As input we used, for each case, a list of stellar absolute magnitudes as derived from a simple theoretical synthetic population (from IACstar, which is available at http://iac-star.iac.es/). We created a complete stellar population down to the lowest mass objects for an assumed star formation history. We have chosen to simulate four generic examples relating to potential science cases:

\footnotetext{
a e-mail: deep@strw.leidenuniv.nl
} 
- An old galaxy at $18 \mathrm{Mpc}$

- An old galaxy at $3 \mathrm{Mpc}$

- A young galaxy at $18 \mathrm{Mpc}$

- A young galaxy at $3 \mathrm{Mpc}$

Though we have done photometry on each of these cases mentioned above, in this paper we present only the most important results.

Table 1. Telescope and instrument parameters.

\begin{tabular}{lll}
\hline Parameter & Unit & Value \\
\hline Collecting area & $\mathrm{m}^{2}$ & 1275 \\
Telescope throughput & & 0.74 \\
AO throughput & 0.80 \\
Instrument throughput & 0.60 \\
Total throughput & 0.40 \\
Readnoise & 5 \\
\hline
\end{tabular}

Table 2. Filter characteristics (Vega magnitudes).

\begin{tabular}{llllll}
\hline Filter & Units & $\mathrm{I}$ & $\mathrm{J}$ & $\mathrm{H}$ & $\mathrm{Ks}$ \\
\hline Filter centre & $\mu \mathrm{m}$ & 0.90 & 1.215 & 1.65 & 2.16 \\
Filter width & $\mu \mathrm{m}$ & 0.24 & 0.26 & 0.29 & 0.32 \\
Zero magnitude & $\mathrm{ph} / \mathrm{s} / \mathrm{m}^{2} / \mu \mathrm{m}$ & $3.76 \mathrm{E}+10$ & $2.02 \mathrm{E}+10$ & $9.56 \mathrm{E}+09$ & $4.66 \mathrm{E}+09$ \\
Sky brightness & $\mathrm{mag} / \mathrm{arcsec}^{2}$ & 19.7 & 16.5 & 14.4 & 13.5 \\
Background & $\mathrm{e}^{-} / \mathrm{s} / \mathrm{pixel}$ & 0.6 & 5.8 & 20.9 & 25.7 \\
\hline
\end{tabular}

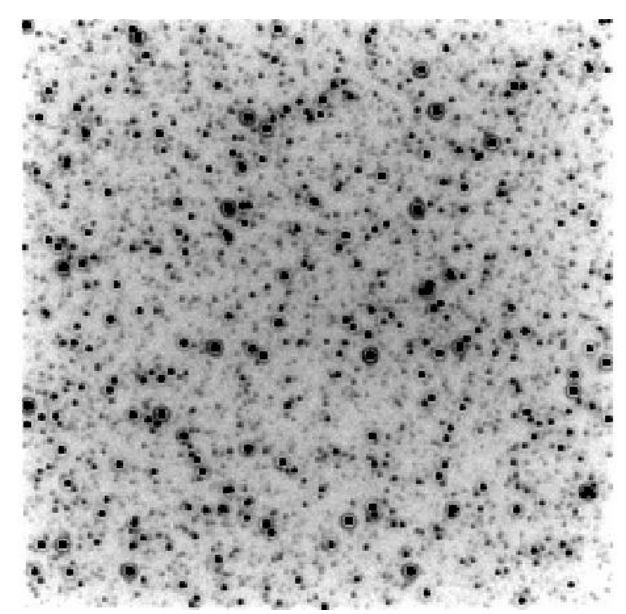

Fig. 1. A simulated MICADO I band image of an old galaxy at $18 \mathrm{Mpc}$ in a field of view of $0.75 \times 0.75$ arcsec $(250 \times 250$ pixels), made using MAORY PSFs. The exposure time is 1 hour. The surface brightness of the galaxy at this position is $\tilde{2} 0 \mathrm{mag} / \operatorname{arcsec}^{2}$ in I band. 
A. Deep et al.: Use of AO PSF models for the Study of Resolved Stellar Populations
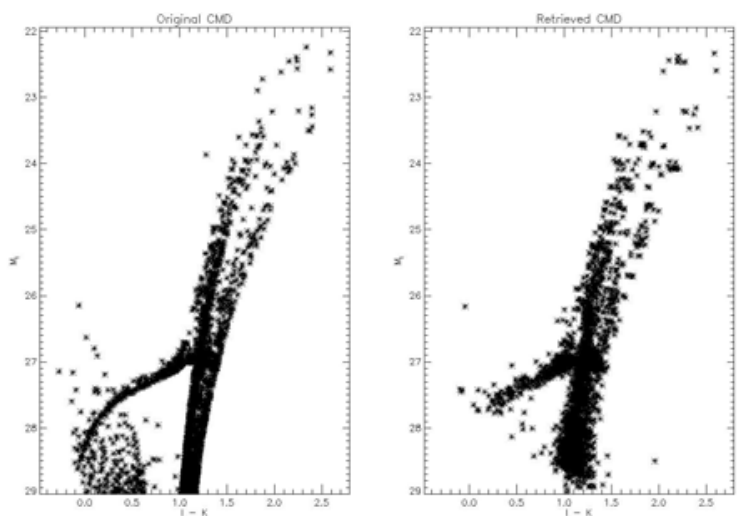

Fig. 2. CMD (I vs. I-Ks) in Vega mag for an old galaxy at $3 \mathrm{Mpc}$ : input (left) and retrieved (right). The Surface brightness is $\mu_{I}=19.9 \mathrm{mag} / \mathrm{arcsec}^{2}$ and $\mu_{K}=18.3 \mathrm{mag} / \mathrm{arcsec}^{2}$. There are 200000 stars in the field of view of 3 $\times 3$ arcsec, going down 7 mags below the detection limit. 14000 stars could be detected using Starfinder.

\section{MCAO}

We have used MAORY PSFs provided to us by the MAORY consortium to simulate the images. Here we pesent results only for an old galxay at a distance of $3 \mathrm{Mpc}$. The Figure 2 contains a pair of CMDs. In the left-hand panel is the input CMD in I and Ks (both Vega magnitudes). In the right-hand panel is the CMD retrieved from the simulated image (measured by Starfinder). To quantify the quality of the photometry we matched the input and retrieved (output) catalogues to each other, and plotted the difference for each filter in the two panels of Figure 3. This is, by definition, the photometric error we achieve. It can be seen that the measured magnitudes do not go deeper than about $\mathrm{I}=29.5 \mathrm{mag}$ for a limiting error of $0.2 \mathrm{mag}$ (which is $20 \%$ photometry or a $5 \sigma$ detection), which is consistent with the magnitude limits predicted in Figure 1. The features in the retrieved CMD are well defined up to this limit, which means that an acceptable photometric accuracy is achieved. As expected, the error becomes steadily larger towards fainter magnitudes. We computed a mean error for each magnitude and these values are over plotted in red on the measurements.
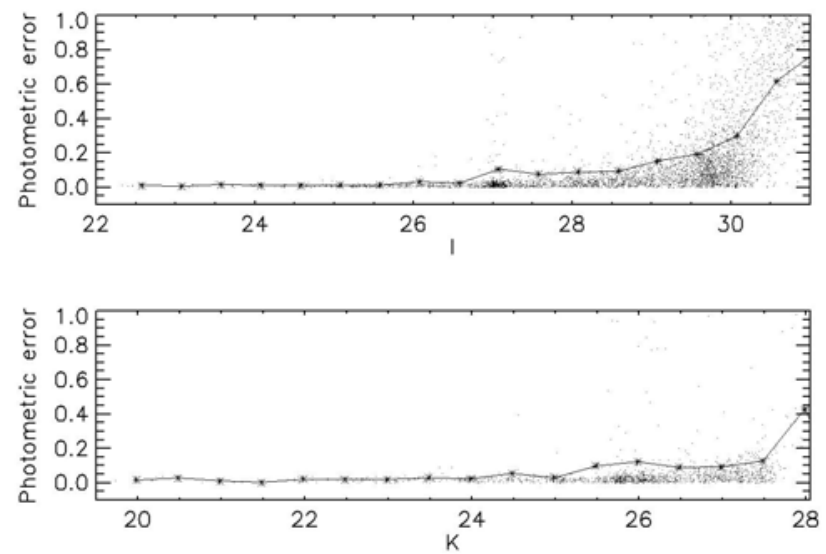

Fig. 3. Difference in magnitudes (photometric error) between the input and output I and Ks catalogues of Figure 9. Over-plotted are RMS errors in bins of $0.5 \mathrm{mag}$. There are 14000 stars in the upper panel and 6000 stars in the lower panel. 


\section{SCAO}

To determine the effect of the elongated PSF on photometric accuracy, we have simulated cases where stars are photometered at distances of 5, 10, 15 and 30 arcsec from the guide star which is at the centre of the field. For the photometry itself, we use only the PSF extracted from a central field. This exercise has been done in both I and Ks filters to compare the impact of anisoplanatism on photometric accuracy at different wavelengths. The resulting uncertainties for the I-band and K-band is shown in Figure 4.

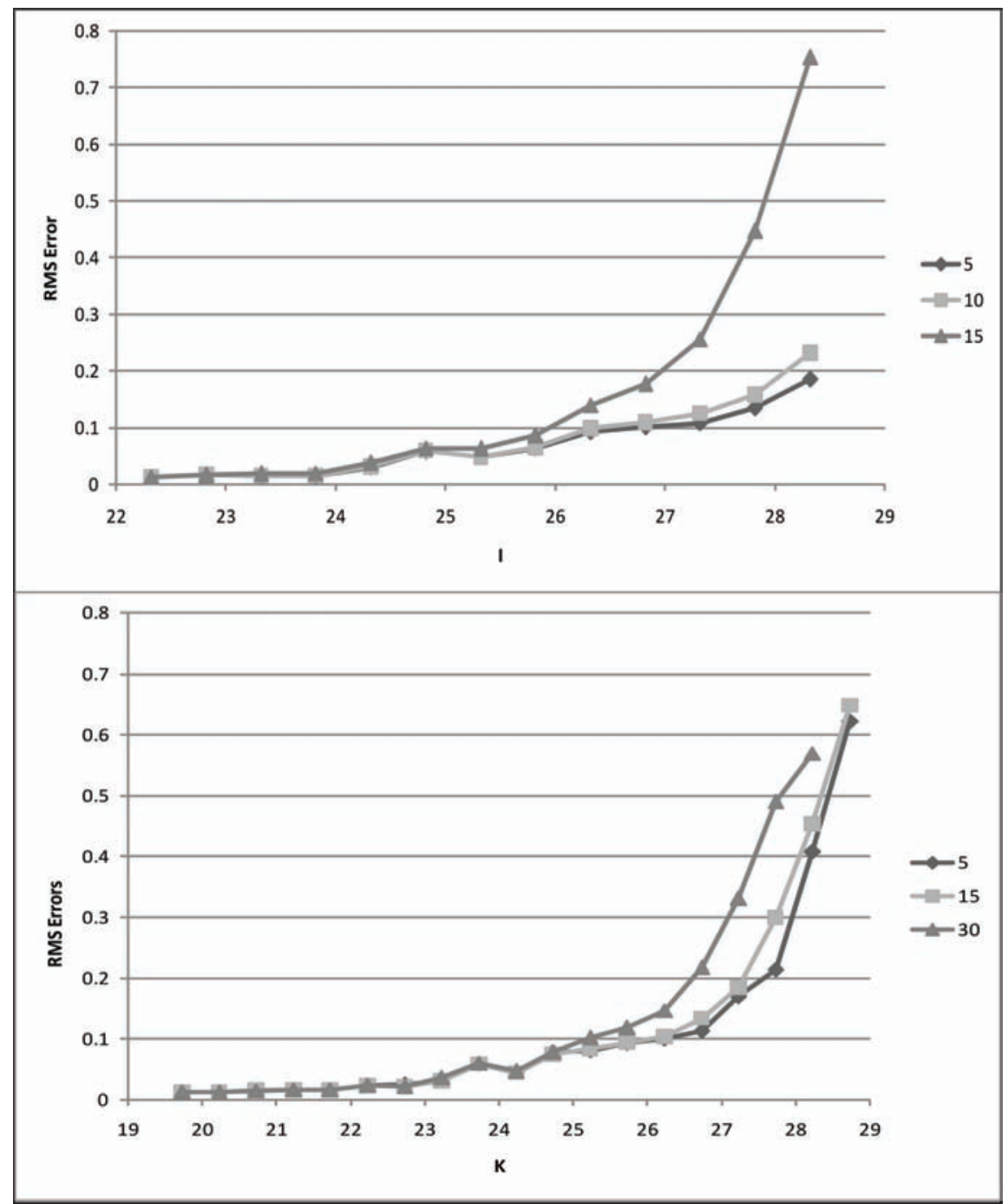

Fig. 4. The effect of anisoplanatism on photometric accuracy in the I (top) and K (bottom) bands. Different lines show the effect of different levels of anisoplanatism when the target is 5, 10, 15 and 30 arcseconds off axis. For $\mathrm{I}$ band, there is no useful information when target is 30 arcseconds off axis.For $\mathrm{K}$ band, 5 an 10 arcseconds lines overlap. 


\section{Effect of crowding}

To measure the effect of different levels of crowding on photometric accuracy, we have performed simulations of MAORY/MICADO that are populated with varying densities of stars. We use as an example the case of an old galaxy at $18 \mathrm{Mpc}$. Putting large numbers of stars into an image is a very computationally intensive task, and so we have simplified the processes by determining the magnitude cut-off below which going deeper only adds to a uniform background. This cut-off has been determined by a trial where we take different cut-offs and see the effect on the fluctuations in the background level. We find that a cut-off 1.5 mag below the detection threshold will have no effect on the background fluctuations because the objects are too faint. To be completely sure we have applied the cut-off at 2 mag below the detection limit. The light from stars fainter than this limit is added to the images as part of a uniform background level which is effectively a sum of their flux.In Figure 5 we show the results of a simulation to study the effect of crowding (i.e. equivalently surface brightness) on stars of a given magnitude in an old galaxy. We look in detail at how accurately the properties of different magnitude stars can be retrieved. The surface brightness in I band varies from $25 \mathrm{mag} / \operatorname{arcsec}^{2}$ to $16 \mathrm{mag} / \operatorname{arcsec}^{2}$. In terms of populated (detected) stars this corresponds to 40 (2000) pixels/star and 0.01(16) pixels/star respectively.

From Figure 5 it can be seen that bright stars (e.g. I=26.3) are almost unaffected by crowding right into the central regions of galaxies where the surface brightness is highest. This is because they are less affected by the background of fainter stars that create most of the crowding. At the other extreme, the faintest stars plotted here $(\mathrm{I}=29.3$ and $\mathrm{I}=30.3)$ are anyway at or beyond the detection limit. But stars which are detected with a reasonable accuracy when isolated $(\mathrm{I}<28)$ can also be probed well into the densest regions that might be expected in Elliptical and Spiral galaxies at the distance of Virgo. Thus we predict that the tip of the Red Giant Branch and evolved AGB stars should be observable at the distance of Virgo with $20 \%$ photometry at a surface brightness $\mu_{I}<16 \mathrm{mag} / \mathrm{arcsec}^{2}$. However, it seems likely that detection of the Horizontal Branch stars in Virgo Elliptical galaxies will only be achievable in the lower surface brightness less crowded outer halo regions.1

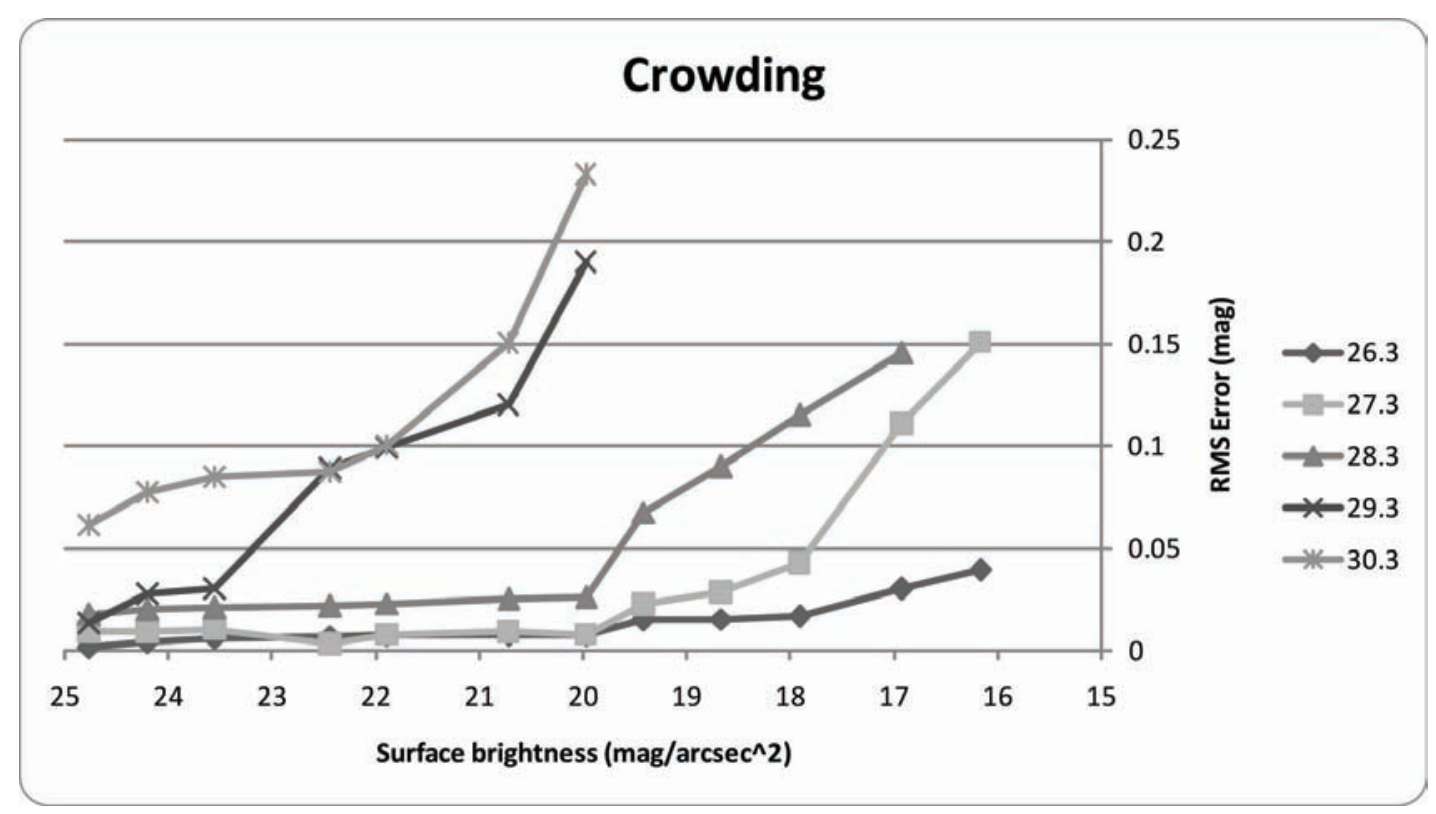

Fig. 5. The effect of crowding for observations in the I-filter for stars of different magnitudes (given to the right) at different surface brightness levels for a galaxy at $18 \mathrm{Mpc}(\mathrm{m}-\mathrm{M}=31.3)$. The number of stars detected ranges from 500 (for $25 \mathrm{mag} / \operatorname{arcsec}^{2}$ ) to $60000\left(16 \mathrm{mag} / \operatorname{arcsec}^{2}\right)$. The stars retrieved go from I=30.3 to I=26.3. 


\section{Summary and conclusions}

In this paper we have addressed the basic issues involved in carrying out accurate photometry in crowded stellar fields for AO images. The conclusions that can be drawn from our study are:

- Standard, publically available, photometry packages such as STARFINDER and DAOPHOT/Allstar do a good job in most circumstances despite the unusually complex shape of the AO PSFs. The CMDs that are measured from the simulated images look very promising and are sufficiently well defined to pick out detailed stellar evolution phases and thus determine the ages of the stars.

- With a SCAO system, good photometry can be obtained over at least 15 arcsec field of view in I and 30 arcsec in Ks-filter. While this is sufficient to obtain a detailed look at distant stellar populations, MCAO has a clear advantage to map resolved stellar populations at the limits of distance and depth in crowded and/or distant galaxies.

- An improvement of the PSF model and more accurate methods of PSF reconstruction (currently carried out by MAORY consortium ) is required to improve the photometric accuracy in very crowded fields in the centres of galaxies at the distance of Virgo.

To summarize, these simulations suggest that it will be possible to carry out deep studies of the resolved stellar populations of Virgo galaxies, going down to the Horizontal Branch in regions of low crowding and potentially detecting the tip of the Red Giant Branch well into the central regions of large Elliptical galaxies.

\section{References}

1. Aparicio A. \& Gallart C., AJ, 128, (2004) 1465

2. Diolaitti, E. et al,. SPIE, 4007, (2000) 879 [Starfinder]

3. Jolissaint, L., Veran, J. P., and Conan, R., JOSA, A 23, (2006/02) 382-394

4. Isen, Blum \& Rigaut, AJ, 126, 452, (2003)

5. MAORY: http://www.bo.astro.it/maory/Maory/

6. Stetson, P. B., PASP, 99,(1987) 191

7. Stetson, P. B. PASP, 106, (1994) 25 